\title{
Brachistochrone of Entanglement for Spin Chains
}

\author{
Alberto Carlini ${ }^{1,2,3}$, 因 and Tatsuhiko Koike ${ }^{4, \dagger}$ \\ ${ }^{1}$ Dipartimento di Scienze ed Innovazione Tecnologica, \\ Universita' del Piemonte Orientale, Alessandria, Italy \\ ${ }^{2}$ Istituto Nazionale di Fisica Nucleare, Sezione di Torino, Gruppo Collegato di Alessandria, Italy \\ ${ }^{3}$ NEST, Istituto di Nanoscienze-CNR, Pisa, Italy \\ ${ }^{4}$ Department of Physics and REC for NS, Keio University, Yokohama, 223-8522, Japan
}

(Dated: December 1, 2016)

\begin{abstract}
We analytically investigate the role of entanglement in time-optimal state evolution as an application of the quantum brachistochrone, a general method for obtaining the optimal time-dependent Hamiltonian for reaching a target quantum state. As a model, we treat two qubits indirectly coupled through an intermediate qubit that is directly controllable, which represents a typical situation in quantum information processing. We find the time-optimal unitary evolution law and quantify residual entanglement by the two-tangle between the indirectly coupled qubits, for all possible sets of initial pure quantum states of a tripartite system. The integrals of the motion of the brachistochrone are determined by fixing the minimal time at which the residual entanglement is maximized. Entanglement plays a role for $W$ and $G H Z$ initial quantum states, and for the bi-separable initial state in which the indirectly coupled qubits have a nonzero value of the 2 -tangle.
\end{abstract}

PACS numbers: 03.67.-a, 03.67.Lx, 03.65.Ca, 02.30.Xx, 02.30.Yy

\section{INTRODUCTION}

The concept of entanglement is one of the key features distinguishing the quantum world from the classical world, as it captures those correlations which cannot have a classical origin [1. It is one of the fundamental resources in quantum information and computation theory (e.g., teleportation [2], superdense coding [3, quantum cryptography [4, quantum state tomography [5], quantum repeaters [6], quantum metrology [7], quantum phase transitions [8], black hole physics 9 etc). Several measures of entanglement have been proposed for multipartite quantum systems in different contexts, for a review see, e.g., 10-12. On the other hand, the speed of evolution for quantum systems is an important concept, not only for determining the theoretical limits at which quantum information can travel 13-[14, but also for the practical task of building quantum computers capable of performing fast quantum algorithms before the ubiquitous and disruptive decoherence effects come into play. The importance of the connection between quantum entanglement, the speed of evolution of quantum systems and dynamical optimization problems has been discussed in [15-21]. Furthermore, quantum optimal control is also a fundamental subject, both theoretically and experimentally, in quantum computing and information (see, e.g., 22] and the road map traced in the recent review [23]). In particular, time-optimal quantum computation, where the cost to be optimized is the time to achieve a given quantum evolution is relevant for the design of fast elementary gates and provides a more physical ground

\footnotetext{
*Electronic address: alberto.carlini@uniupo.it
}

${ }^{\dagger}$ Electronic address: koike@phys.keio.ac.jp to describe the complexity of quantum algorithms. A theoretical framework for the quantum brachistochrone (QB) was introduced in 24]. The QB [25] is based on a variational principle enforcing the time-optimal evolution of a quantum system whose Hamiltonian is subject to a set of constraints (e.g., a finite energy, certain qubit interactions are forbidden etc..) and defines a boundary value problem with fixed initial and final quantum states (or unitary transformations). The QB has been studied for quantum state evolution in the case of pure 24] and mixed states 26, for the optimal realization of unitary transformations up to a given target quantum gate [27, for the more realistic situation where the target can be reached within a finite, tolerable error (a fixed fidelity) 28, and for the time optimal transfer of coherence in a trilinear Ising spin chain 29]. An efficient numerical algorithm for the QB was proposed and applied for finding evidence that the $\mathrm{QB}$ can be used to estimate the gate complexity of unitary operators 30. It was also shown 31] that the $\mathrm{QB}$ can be recast as the problem of finding geodesics in a suitable Hilbert space and can be used to solve the famous Zermelo navigation problem via the aid of a Finslerian geometry 32 . Very recently 33 experimental time optimal universal control of spin qubits from diamonds with NV centers has been finally demonstrated based on the QB formalism. On the other hand, the engineering of the generation and the dynamics, including the effects of (sudden) death and revival of tripartite entanglement for the prototypical $W$ or $G H Z$ states has been widely studied, e.g. with QED in optical cavities under the action of Markovian or non Markovian noise in [34 - 39], or within generic three qubit systems, e.g. [40] - 45]. Fast and robust engineering of tripartite entanglement from the vibrational modes in optomechanical systems can be found in 46, while the generation of $W$ and $G H Z$ states has been studied, e.g., with the 
technique of shortcut to adiabatic passage, both using dressed states with SQUID qubits [4] or atoms in cavity QED [48. The dynamics of tripartite entanglement has been recently studied even in the context of gravity for accelerated qubits coupled to a real scalar field in the vicinity of black holes [49]-[50], and for multiple identical fermions undergoing decoherence [51. Quasi local control protocols for a dissipative engineering of $G H Z$ and $W$ states can be found in, e.g., 52, while the generation and stabilisation of the same states in superconducting circuit $\mathrm{QED}$ via quantum feedback control techniques is shown in [53. Finally, optimal control methods to generate entanglement have been recently applied to continuous variable systems, e.g., for two mode systems in linear networks 54 or for two mode Gaussian states subject to Ohmic relaxation [55], for plasmonically coupled quantum dots in cavity QED [56] and for noise resistant spinsqueezing in strongly interacting many body systems [57]. Recently, some authors [58-59] started the study of the role of quantum entanglement during the QB evolution of multipartite distinguishable systems in a pure quantum state, finding that the entanglement is pivotal to the QB evolution if at least two subsystems actively evolve. Efficient generation of random multipartite entangled states has been also analyzed with the aid of time-optimal unitary operations 60. More recently, the authors of 61. found that genuine tripartite entanglement is necessary during the $\mathrm{QB}$ evolution of a set of three qubits in the pure state, except for the case in which less than three qubits attend evolution.

In this paper, we discuss the role played by entanglement in time-optimal state evolution, as an application of the QB formalism. Though our method can be applied to general systems, we consider a simple concrete model where two qubits are coupled via an intermediate qubit which can be controlled directly. This is a typical situation in a wide array of promising (scalable) experimental realizations of quantum information processing (see, e.g., 62-68). For example, the system can be considered as a spin chain under on-site magnetic fields. An advantage of the model is that one can analytically work out all the details including the time evolution of the state and it entanglement. Moreover, the QB formalism naturally allows for the situation in which local coherent controls are assumed to be time consuming, contrary to the requirements of zero time cost for local controls typical of the standard time-optimal quantum control methods [69- 70]. We concentrate our attention on the behavior of the residual entanglement between the indirectly coupled qubits at the end of a trilinear chain, as expressed by the so called 2-tangle [71. We also consider all the possible initial quantum pure states for the tripartite system, i.e. completely separable, bi-separable, and with true tripartite entanglement (fully bipartite for $W$ and maximally tripartite for $G H Z$ states). We then let the 2-tangle evolve along the general time-optimal quantum trajectory defined by the QB action principle, and we fix the integrals of the motion by imposing that entanglement reaches its maximum value in the shortest possible time. The minimal time for reaching such a maximum of the 2-tangle is a function of the ratio between the interaction couplings of the Ising Hamiltonian.

The paper is organized as follows. In Section II we review the main features of the QB formalism for the time-optimal synthesis of unitary quantum evolutions. In Section III we summarize the QB solution for the problem of a three-linear qubit system subject to an Ising interaction with unequal couplings and a local control on the intermediate qubit, when a finite energy is available. In Section IV we define the measure for the bipartite entanglement between the two indirectly coupled qubits of the chain and we introduce the main formulas for the 2-tangle and 3-tangle for all the possible sets of initial quantum states. Section V is devoted to the study of the entanglement evolution for these initial quantum states, and we define the optimal times and the analytical form of the quantum evolutions for which the 2-tangle between the indirectly coupled qubits most rapidly reach its maximum value. Finally, Section IV is devoted to the summary and discussion of our results.

\section{TIME-OPTIMAL UNITARY EVOLUTION}

The goal is to determine the time-optimal way to generate the unitary evolution up to a certain $U_{f}$ (modulo physically irrelevant overall phases) by controlling an Hamiltonian $H(t)$ obeying the Schrödinger equation. We assume that $H$ is controllable and that only a finite energy is available in the experiment. This time-optimality problem may be formulated using the action [27]:

$$
\begin{aligned}
S\left(U, H ; \alpha, \Lambda, \lambda_{j}\right) & :=\int_{0}^{1} d s\left[N \alpha+L_{S}+L_{C}\right], \\
L_{S} & :=\left\langle\Lambda, i \frac{d U}{d s} U^{\dagger}-\alpha H\right\rangle, \\
L_{C} & :=\alpha \sum_{j} \lambda_{j} f^{j}(H),
\end{aligned}
$$

where $\langle A, B\rangle:=\operatorname{Tr}\left(A^{\dagger} B\right)$ and the Hermitian operator $\Lambda(s)$ and the real functions $\lambda_{j}(s)$ are Lagrange multipliers. The quantity $\alpha$ is the time cost, relating the parameter time $s$ and the physical time $t$ via $t:=\int \alpha(s) d s$. Variation of $L_{S}$ by $\Lambda$ gives the Schrödinger equation:

$$
i \frac{d U}{d t}=H U, \quad \text { or } \quad U(t)=\mathcal{T} e^{-i \int_{0}^{t} H d t},
$$

where $\mathcal{T}$ is the time ordered product. Variation of $L_{C}$ by $\lambda_{j}$ leads to the constraints for $H$ :

$$
f_{j}(H)=0 .
$$

In particular, the finite energy condition for a system of $\log N$ qubits reads:

$$
f_{0}(H):=\frac{1}{2}\left[\operatorname{Tr}\left(H^{2}\right)-N \omega^{2}\right]=0,
$$


where $\omega$ is a constant.

From the variation of $S$ with respect to $H$ we get:

$$
\Lambda=\lambda_{0} H+\sum_{j \neq 0} \lambda_{j} \frac{\partial f_{j}(H)}{\partial H},
$$

while from the variation of $S$ by $\alpha$ we obtain the normalization condition:

$$
\operatorname{Tr}(H \Lambda)=N
$$

Finally, variation of $S$ by $U$, use of eq. (7) and some elementary algebra give the quantum brachistochrone equation:

$$
i \frac{d \Lambda}{d t}=[H, \Lambda]
$$

The quantum brachistochrone (9) together with the constraints (5) define a boundary-value problem for the evolution of the unitary operator $U(t)$ with fixed initial $(U(t=0)=1$, where 1 is the identity matrix) and final conditions $\left(U(t=T)=U_{f}\right.$, where $T$ is the optimal time duration necessary to achieve the target gate $\left.U_{f}\right)$. It can be solved together with the constraint functions $f_{j}(H)$ to obtain $H_{\text {opt }}(t)$. Then one integrates the Schrödinger equation (4) with $U(0)=1$ to get $U_{\text {opt }}(t)$ and finally the integration constants in $H_{\mathrm{opt}}(t)$ can be fixed, e.g., by imposing that $U_{\text {opt }}(T)$ equals a target $U_{f}$ modulo a global (physically irrelevant) phase.

\section{QB FOR A TRILINEAR ISING CHAIN}

We now apply the general formalism of the QB to the case of a physical system of three qubits (labeled by a superscript $a \in\{1,2,3\})$ interacting via an Ising Hamiltonian and where the intermediate qubit is subject to a local and controllable magnetic field $B_{i}(t)(i=x, y, z)$ :

$$
H(t):=J_{12} \sigma_{z}^{1} \sigma_{z}^{2}+J_{23} \sigma_{z}^{2} \sigma_{z}^{3}+\vec{B}(t) \cdot \vec{\sigma}^{2},
$$

where we have defined $\sigma_{i}^{1} \sigma_{j}^{2}:=\sigma_{i} \otimes \sigma_{j} \otimes 1, \sigma_{i}^{2} \sigma_{j}^{3}:=$ $1 \otimes \sigma_{i} \otimes \sigma_{j}, \sigma_{i}^{2}:=1 \otimes \sigma_{i} \otimes 1$ and $\sigma_{i}$ are the Pauli operators. Introducing the ratio between the Ising interaction couplings $K:=J_{23} / J_{12}$ and rescaling time as $\tau:=J_{12} t$, the energy as $\hat{\omega}:=\omega / J_{12}$ and the magnetic field as $\vec{B}_{\text {opt }}(t)=J_{12} \overrightarrow{\hat{B}}_{\text {opt }}(\tau)$, it can be shown [28] that the $\mathrm{QB}$ is solved by the following time-optimal magnetic field:

$$
\overrightarrow{\hat{B}}_{\mathrm{opt}}(\tau)=\left(\begin{array}{c}
\hat{B}_{0} \cos \theta(\tau) \\
\hat{B}_{0} \sin \theta(\tau) \\
\hat{B}_{z}
\end{array}\right)
$$

where $\theta(\tau):=\hat{\Omega} \tau+\theta_{0}$ and $\hat{\Omega}, \theta_{0}, \hat{B}_{0}$ and $\hat{B}_{z}$ are integration constants. The magnetic field is precessing around the $z$-axis with the frequency $\hat{\Omega}$. Furthermore, the energy constraint explicitly reads $\overrightarrow{\hat{B}}^{2}=\hat{\omega}^{2}-\left(1+K^{2}\right):=\hat{\omega}_{K}^{2}$, so that we can reparameterize:

$$
\hat{B}_{0}:=\hat{\omega}_{K} \cos \phi ; \quad \hat{B}_{z}:=\hat{\omega}_{K} \sin \phi,
$$

where and $\phi \in[0,2 \pi]$.

One can then solve the Schrödinger equation (4) and find the time-optimal evolution operator (for more details, see Appendix B of [28]):

$$
\begin{aligned}
U_{\mathrm{opt}}(\tau) & =e^{-i \frac{\hat{\Omega} \tau}{2}} A_{D}^{13-}(\tau)|0\rangle_{2}\left\langle 0\left|+e^{i \frac{\hat{\Omega} \tau}{2}} A_{D}^{13+}(\tau)\right| 1\right\rangle_{2}\langle 1| \\
& -i B_{0} S_{D}^{13}(\tau)\left[e^{i\left(\frac{\hat{\Omega} \tau}{2}-\theta(\tau)\right)}|0\rangle_{2}\langle 1|+\text { H.c. }\right],
\end{aligned}
$$

where we have introduced the diagonal operators acting in the Hilbert space of qubits 1 and 3:

$$
\begin{aligned}
A_{D}^{13 \pm}(\tau) & :=\operatorname{Diag}\left[\alpha_{1}^{ \pm}(\tau), \alpha_{2}^{ \pm}(\tau), \alpha_{3}^{ \pm}(\tau), \alpha_{4}^{ \pm}(\tau)\right], \\
S_{D}^{13}(\tau) & :=\operatorname{Diag}\left[s_{1}(\tau), s_{2}(\tau), s_{3}(\tau), s_{4}(\tau)\right] .
\end{aligned}
$$

The latter operators depend upon the functions of time:

$$
\begin{aligned}
s_{i}(\tau) & :=\frac{\sin \left(\omega_{i} \tau\right)}{\omega_{i}}, \\
c_{i}(\tau) & :=\cos \left(\omega_{i} \tau\right), \\
\alpha_{i}^{ \pm}(\tau) & :=c_{i}(\tau) \pm i b_{i} s_{i}(\tau),
\end{aligned}
$$

and on the constants:

$$
\begin{aligned}
\omega_{i} & :=\hat{\omega}_{K} \sqrt{\cos ^{2} \phi+b_{i}^{2}}, \\
b_{i} & :=\sin \phi+\frac{1}{\hat{\omega}_{K}}\left[\left(\delta_{i 1}-\delta_{i 4}\right)(1+K)\right. \\
& \left.+\left(\delta_{i 2}-\delta_{i 3}\right)(1-K)-\frac{\hat{\Omega}}{2}\right],
\end{aligned}
$$

where $i=1,2,3,4$ and $\delta_{i j}$ is the Kronecker symbol.

\section{ENTANGLEMENT IN THE 1-3 SUBSYSTEM}

In this section, we start the analysis of the role played by the entanglement in time-optimal quantum state evolutions. We consider the time-optimal evolution of an arbitrary initial pure state $|\psi(0)\rangle$ driven by the unitary operator (13) and study the behavior of the bipartite entanglement between indirectly coupled qubits 1 and 3 . We are interested, in particular, in determining the optimal time $\tau_{*}$ and the integration constants $\hat{\Omega}, \theta_{0}, \phi$ for which the the entanglement between qubits 1 and 3 is maximized.

A pure state of a tripartite quantum system, with each party being a qubit, can be written as:

$$
|\psi\rangle=\sum_{i=0}^{7} a_{i}|i\rangle
$$


where $|0\rangle:=|000\rangle,|1\rangle:=|001\rangle,|2\rangle:=|010\rangle,|3\rangle:=|011\rangle$, $|4\rangle:=|100\rangle,|5\rangle:=|101\rangle,|6\rangle:=|110\rangle,|7\rangle:=|111\rangle$. The entanglement between two of its subsystems, e.g. 1 and 3, may be quantified by the 2-tangle [9], [71]:

$$
\tau_{13}:=2\left(\operatorname{Det}\left[\rho_{1}\right]-\operatorname{Det}\left[\rho_{2}\right]+\operatorname{Det}\left[\rho_{3}\right]-|\operatorname{HypDet}(a)|\right),
$$

where $\rho_{i}(i=1,2,3)$ are the reduced density matrices:

$$
\begin{aligned}
& \rho_{1}:=\operatorname{Tr}_{23}(|\psi\rangle\langle\psi) \mid), \\
& \rho_{2}:=\operatorname{Tr}_{13}(|\psi\rangle\langle\psi) \mid), \\
& \rho_{3}:=\operatorname{Tr}_{12}(|\psi\rangle\langle\psi) \mid),
\end{aligned}
$$

and HypDet $(a)$ is Cailey's hyperdeterminant for the matrix of the coefficients $a_{i}$ s (see eq. (47) in the Appendix).

One may also define the 3-tangle:

$$
\tau_{123}:=|\operatorname{HypDet}(a)|
$$

which describes the amount of tripartite entanglement between all the spins.

The pure states of a tripartite quantum system can be classified into equivalence classes under local operations and classical communication (LOCC), which are distinguished by the degree of entanglement between its subsystems [2]. In particular, we will consider the representatives of each of these classes as a possible initial quantum state, i.e.:

S) completely separable states, for which all 2-tangles and the 3-tangle vanish:

$$
|\psi(0)\rangle=\left|\psi_{S}\right\rangle:=|000\rangle
$$

B) bi-separable states, with bipartite entanglement, for which one of the 2-tangles is nonzero while the other 2tangles and the 3 -tangle are zero:

$$
\begin{aligned}
& |\psi(0)\rangle=\left|\psi_{B 1}\right\rangle:=\frac{1}{\sqrt{2}}(|001\rangle+|010\rangle), \\
& |\psi(0)\rangle=\left|\psi_{B 2}\right\rangle:=\frac{1}{\sqrt{2}}(|001\rangle+|100\rangle), \\
& |\psi(0)\rangle=\left|\psi_{B 3}\right\rangle:=\frac{1}{\sqrt{2}}(|010\rangle+|100\rangle) ;
\end{aligned}
$$

W) W states, with full bipartite entanglement, for which all 2-tangles are non zero while the 3-tangle vanishes:

$$
|\psi(0)\rangle=\left|\psi_{W}\right\rangle:=\frac{1}{\sqrt{3}}(|001\rangle+|010\rangle+|100\rangle)
$$

GHZ) GHZ states with maximal tripartite entanglement, for which all 2-tangles and the 3-tangle are nonzero:

$$
|\psi(0)\rangle=\left|\psi_{G H Z}\right\rangle:=\frac{1}{\sqrt{2}}(|000\rangle+|111\rangle) .
$$

The time-optimal evolution operator $U_{\text {opt }}(\tau)$, eq. (13), drives the initial states according to:

$$
|\psi(\tau)\rangle=U_{\text {opt }}(\tau)|\psi(0)\rangle=\sum_{i=0}^{7} a_{i}(\tau)|i\rangle .
$$

For each of the possible classes of initial states, one can calculate the tangles $\tau_{13}(\tau)$ and $\tau_{123}(\tau)$ exactly. Namely, one computes the time-dependent amplitudes $a_{i}(\tau)$ through $(30)$, whose nonvanishing values are given by formulas (52)-(57) in the Appendix, and substitutes these formulas into (47) and (51), and then into (21) and (23).

We obtained the following results.

S-B1-B3) When there is no entanglement between the indirectly coupled qubits 1 and 3 initially, i.e. when the state $|\psi(0)\rangle$ is fully separable or biseparable of type $B 1$ or $B 3$, both the $\tau_{13}(\tau)$ and the $\tau_{123}(\tau)$ tangles are always zero during the whole time-optimal evolution.

On the other hand, when there is some initial entanglement between qubits 1 and 3, then we have a non trivial time-optimal evolution of the $\tau_{13}(\tau)$ and $\tau_{123}(\tau)$ tangles.

B2) When the initial state belongs to the class $B 2$ in eq. 26) of bi-separable states, from eqs. (13), (21), (23), (30), (47), (51) and (54) and we get the following timeoptimal evolutions:

$$
\begin{aligned}
\tau_{13_{B 2}}(\tau) & =\left|a_{2}^{*} a_{3}+\hat{B}_{0}^{2} s_{2} s_{3}\right|^{2}, \\
\tau_{123_{B 2}}(\tau) & =\frac{\hat{B}_{0}^{2}}{4}\left|a_{2} s_{3}-a_{3} s_{2}\right|^{2}=1-\tau_{13_{B 2}}(\tau) .
\end{aligned}
$$

W) The case of an initial $W$ state is similar and we find that $\tau_{13_{W}}(\tau)=(4 / 9) \tau_{13_{B 2}}(\tau)$ and $\tau_{123_{W}}(\tau)=$ $(4 / 9) \tau_{123_{B 2}}(\tau)$.

GHZ) Finally, for the class of fragile, fully entangled $G H Z$ states, the time-optimal evolution of the tangles is found to be:

$$
\begin{aligned}
\tau_{13_{G H Z}}(\tau) & =\hat{B}_{0}^{2}\left|a_{1} s_{4}-a_{4} s_{1}\right|^{2}, \\
\tau_{123_{G H} Z}(\tau) & =\frac{1}{4}\left|a_{1}^{*} a_{4}+\hat{B}_{0}^{2} s_{1} s_{4}\right|^{2}=1-\tau_{13_{G H Z}}(\tau) .
\end{aligned}
$$

\section{TIME-OPTIMAL EVOLUTION OF ENTANGLEMENT}

In this section, we shall study in more detail the behavior of the time-optimal evolution of the tangles $\tau_{13}$ and $\tau_{123}$ which witness the entanglement between qubits 1 and 3 .

We use of the formulas $16,(18)$ and $(19)$ explicitly in equations (31)-(34). As the tangles are always related by $\tau_{13}(\tau)=1-\tau_{123}(\tau)$, we limit ourselves to the study of the $\tau_{13}$ tangle.

B2-W) For the case of an initial bi-separable state of class $B 2$ (and similarly for initial states of the class $W$ ), from (18) and (31) we obtain:

$$
\begin{aligned}
\tau_{13_{B 2}}(\tau) & =\left[c_{2} c_{3}+\hat{\omega}_{K}^{2}\left(\cos ^{2} \phi+b_{2} b_{3}\right) s_{2} s_{3}\right]^{2} \\
& +\hat{\omega}_{K}^{2}\left(b_{2} s_{2} c_{3}-b_{3} s_{3} c_{2}\right)^{2} .
\end{aligned}
$$


We now proceed by further optimizing the tangle $\tau_{13_{B 2}}(\tau)$ as a function of the time $\tau$ and of the unknown constants of the motion for the time-optimal quantum trajectory, i.e. $\hat{B}_{0}, \hat{B}_{z}, \hat{\Omega}$, for a given ratio of the couplings $K$ and for fixed energy $\hat{\omega}$. In particular, we look for the time $\tau_{*}$ at which the tangle first reaches its maximum value $\left.\tau_{13_{B 2}}\right|_{\max }=1$ by imposing that the partial derivatives of the tangle with respect to $\tau$ and the other constants of the motion (expressed in terms of $\phi$ and $\hat{\Omega}$ ) vanish and that the determinant of the Hessian matrix is negative. After a long and tedious but simple algebra we find that "optimal" time at which $\tau_{13_{B 2}}(\tau)$ first reaches its maximum depends on the value of the available energy $\hat{\omega}^{2}$ and on the ratio $K$ between the couplings in the Ising Hamiltonian. In more details, we have that:

$$
\tau_{*_{B 2}}=\frac{\sqrt{3}}{4} \frac{\pi}{|1-K|},
$$

when $1<\hat{\omega}^{2}<29 / 16$ and $|K|<K_{1+}$, or when $\hat{\omega}^{2}>29 / 16$ and $K_{1-}<K<K_{2-}$ or $K_{2+}<K<$ $K_{1+}$, where we have defined $K_{1 \pm}:= \pm \sqrt{\hat{\omega}^{2}-1}$ and $K_{2 \pm}:=(1 / 4)\left[13 / 4 \pm \sqrt{3\left(\hat{\omega}^{2}-29 / 16\right)}\right]$. The duration (36) of the optimal quantum evolution for the bi-partite entanglement is minimal for a ratio of the Ising couplings $K \rightarrow \mp K_{1-}$, and maximal for a ratio of the Ising couplings $K \rightarrow K_{1+}$. Furthermore, in this case we obtain the optimal magnitudes and frequency for the magnetic field as:

$$
\begin{aligned}
\left|\hat{B}_{0_{B 2}}\right| & =\frac{2}{\sqrt{3}}|K-1|, \\
\left|\hat{B}_{z_{B 2}}\right| & =\sqrt{\hat{\omega}^{2}-\frac{7}{3} K^{2}+\frac{8}{3} K-\frac{49}{21}}, \\
\hat{\Omega}_{B 2} & =2\left[K-1 \pm \sqrt{\hat{\omega}^{2}-\frac{7}{3} K^{2}+\frac{8}{3} K-\frac{49}{21}}\right] .
\end{aligned}
$$

Instead, we have that:

$$
\tau_{*_{B 2}}=\frac{\pi}{\sqrt{\hat{\omega}^{2}-2 K}},
$$

when $\hat{\omega}^{2}>29 / 16$ and $K_{2-}<K<K_{2+}$. Within this range of allowed energies and $K$, the duration (40) of the optimal quantum evolution for the bi-partite entanglement is minimal for a ratio of the Ising couplings $K \rightarrow K_{2-}$, and maximal for a ratio of the Ising couplings $K \rightarrow K_{2+}$. In this case, the optimal values for the magnetic field are:

$$
\begin{aligned}
& \left|\hat{B}_{0_{B 2}}\right|=\left|\hat{\omega}_{K}\right|, \\
& \left|\hat{B}_{z_{B 2}}\right|=|\hat{\Omega}|=0 .
\end{aligned}
$$

In Figs. 1-2 we plot the 2-tangle and the 3-tangle as a function of time when the quantum system is in the initial bi-separable state $B 2$ and for the value of energy $\hat{\omega}=\sqrt{6}$. For this value of energy, we can compute $K_{1 \pm}= \pm 2.24$ and $K_{2+} \simeq 1.70, K_{2-} \simeq-0.007$. To exemplify the values of the couplings, we consider two models proposed in [66] for nuclear magnetic resonance (NMR) experiments, i.e.: i) the $H-N-H$ chain in the molecule of ethanamide, for which $J_{12}=J_{23} \simeq 88.05 \mathrm{~Hz}$ and therefore $K=1$; ii) the $P-F-H$ chain in the molecule of diethylfluoromethylphosphonate, for which $J_{12} \simeq 46 \mathrm{~Hz}, J_{23} \simeq 73.1 \mathrm{~Hz}$ and therefore $K=1.59$. For both $K=1$ and $K=1.59$ we are in the situation depicted by formula (40), and the law of quantum evolution of the tangle is explicitly given by $\tau_{13_{B 2}}(\tau)=$ $1-4\left[(1-K)^{2} \hat{\omega}_{K}^{2} /\left(\hat{\omega}^{2}-2\right)^{2}\right] \sin ^{4}\left[\sqrt{\hat{\omega}^{2}-2 K} \tau\right]$. We notice that the case of equal Ising couplings, i.e. case i) with $K=1$, residual entanglement between the indirectly coupled qubits is constant and always maximal, equal to one, while the tripartite entanglement is always zero. For the case ii) of unequal couplings, instead, the bipartite entanglement has a periodic behavior, starting from the maximum equal to one at $\tau=0$, reaching a minimum of approximately 0.57 at $\tau=\tau_{*_{B 2}} / 2$, rising again to the maximum of one at $\tau=\tau_{*_{B 2}}$ and so on.

GHZ) Let us now turn to the case of an initial state belonging to the $G H Z$ class. From (18) and (33) we obtain:

$$
\begin{aligned}
\tau_{13_{G H Z}}(\tau) & =\hat{\omega}_{K}^{2} \cos ^{2} \phi\left[\left(c_{1} s_{4}-c_{4} s_{1}\right)^{2}\right. \\
& \left.+\hat{\omega}_{K}^{2}\left(b_{4}-b_{1}\right)^{2}\left(s_{1} s_{4}\right)^{2}\right] .
\end{aligned}
$$

In this case, the "optimal" time at which $\tau_{13_{G H Z}}(\tau)$ first reaches its maximum is found to be:

$$
\tau_{*_{G H Z}}=\frac{\sqrt{2}}{4} \frac{\pi}{|1+K|},
$$

provided that $\hat{\omega}^{2}>3 / 2$ and $K_{-}<K<K_{+}$, where we have defined $K_{ \pm}:=(1 / 2)\left[-1 \pm \sqrt{2 \hat{\omega}^{2}-3}\right]$. For the $G H Z$ initial states, the duration (44) of the optimal quantum evolution for the bi-partite entanglement is minimal for a ratio of the Ising couplings $K \rightarrow K_{+}$, and maximal for a ratio of the Ising couplings $K \rightarrow K_{-}$(and it diverges if $\hat{\omega}^{2}=2$, and $K \rightarrow K_{-}=-1$ ). The associated optimal magnitudes and frequency for the magnetic field in the $G H Z$ case are:

$$
\begin{aligned}
& \left|\hat{B}_{0_{G H Z}}\right|=|1+K|, \\
& \left|\hat{B}_{z_{G H}}\right|=\frac{\left|\hat{\Omega}_{G H Z}\right|}{2}=\sqrt{\hat{\omega}^{2}-2\left(K^{2}+K+1\right)} .
\end{aligned}
$$

In Figs. 3-4 we plot the 2-tangle and the 3-tangle as a function of time when the quantum system is in the initial $G H Z$ state and for the value of energy $\hat{\omega}=\sqrt{14}$. For this energy we have that $K_{-}=-3$ and $K_{+}=2$. Therefore, we can still use the NMR models i) and ii) of Figs. 1-2, with $K=1$ and $K=1.59$, respectively. Here the explicit analytical formula for the time-optimal evolution of the entanglement is given by $\tau_{13_{G H}}(\tau)=\sin ^{4}[\sqrt{2}(1+K) \tau]$. Now the periodical behavior is present for both values of $K$, and the system oscillates between the initial maximal bipartite entanglement and zero entanglement. 


\section{DISCUSSION}

We have analytically investigated the problem of the time-optimal unitary evolution of (tripartite) entanglement, a fundamental resource in quantum computation and quantum information [11. How to robustly create entanglement in the shortest possible way (to fight decoherence etc...) is a crucial task which is the subject of several efforts in the literature (see Introduction). Our method of analysis based on the quantum brachistochrone $(\mathrm{QB})$, developed by the present authors, is very general and can be applied to arbitrary quantum systems. The model that we considered consists of indirectly coupled qubits via an intermediate qubit that is directly controllable. An example of a concrete physical system which realizes the model is a trilinear Ising chain with unequal interaction couplings, with the middle spin controlled by a local magnetic field. The entanglement is quantified by the 2-tangle between the two qubits at the end of the chain. Using the formalism of the QB with the constraint of a fixed energy available, we have analytically found the time-optimal unitary evolution law for the Ising Hamiltonian plus the local control and we substituted it in the formula for the 2-tangle. The initial boundary condition for the QB is chosen among all possible sets of tripartite quantum pure states, i.e. fully separable, bi-separable, $W$ and $G H Z$ states. The integrals of the motion in the QB are determined imposing that the 2-tangle reaches its maximum in the shortest time possible, which we call $\tau_{*}$. Entanglement is found to have a non trivial role during the time-optimal unitary evolutions of $W$ and $G H Z$ initial quantum states, and of the bi-separable initial state in which the indirectly coupled qubits have a nonzero value of the 2 -tangle. The optimal time $\tau_{*}$ also sets the time-scale for the duration of the significant role of the entanglement, and it is a function of the ratio $K$ between the interaction couplings in the Ising Hamiltonian and of the energy available in the experiment. The monogamy of entanglement shows neatly in the anti-correlation of the tripartite entanglement, quantified by the 3 -tangle, with the bipartite entanglement, quantified by the 2-tangle. The QB method has been used to study a physical example which is a typical and interesting scenario in quantum information processing and which had been investigated under different perspectives in the previous literature [62]-68]. We extended this investigation to the important case of entanglement, and our work is a rare example where this kind of analysis is done in a completely analytical manner. It is well known in the theory of quantum optimal control that going beyond and considering the analytical description of more complex (e.g., with more than 2-3 qubit) systems is an extremely challenging task (though several results on higher dimensional models exist, they are all based on numerical approaches). Nevertheless, it is our intention to try and extend the analysis presented here at least to the case when, e.g., coherent control is possible on all the qubits in the chain, to longer chains of qubits (with certain symmetries), and to study the QB evolution of the truly non classical correlations via their proper measure, quantum discord [73].

\section{ACKNOWLEDGEMENTS}

A.C. acknowledges the support from the MIUR of Italy under the program "Rientro dei Cervelli". T.K acknowledges the support from MEXT-Supported Program for the Strategic Research Foundation at Private Universities "Topological Science" and from Keio University GSEC Creativity Initiative "Quantum Community".

\section{APPENDIX}

For a tripartite system 123 in a pure state $|\psi\rangle=$ $\sum_{i=0}^{7} a_{i}|i\rangle$ expanded in the basis $\{|0\rangle:=|000\rangle,|1\rangle:=$ $|001\rangle,|2\rangle:=|010\rangle,|3\rangle:=|011\rangle,|4\rangle:=|100\rangle,|5\rangle:=|101\rangle$, $|6\rangle:=|110\rangle,|7\rangle:=|111\rangle\}$, Cailey's hyperdeterminant for the matrix of the coefficients $a_{i} \mathrm{~s}$ is defined as:

$$
\begin{aligned}
\operatorname{HypDet}(a) & :=\left[\left(a_{0} a_{7}\right)^{2}+\left(a_{1} a_{6}\right)^{2}+\left(a_{2} a_{5}\right)^{2}+\left(a_{3} a_{4}\right)^{2}\right] \\
& -2\left[\left(a_{0} a_{7}+a_{1} a_{6}\right)\left(a_{2} a_{5}+a_{3} a_{4}\right)\right. \\
& \left.+a_{0} a_{1} a_{6} a_{7}+a_{2} a_{3} a_{4} a_{5}\right] \\
& +4\left(a_{0} a_{3} a_{5} a_{6}+a_{1} a_{2} a_{4} a_{7}\right) .
\end{aligned}
$$

More explicitly, since:

$$
\begin{aligned}
\operatorname{Det}\left(\rho_{1}\right) & =\left|a_{0}\right|^{2}\left(\left|a_{5}\right|^{2}+\left|a_{6}\right|^{2}+\left|a_{7}\right|^{2}\right) \\
& +\left|a_{1}\right|^{2}\left(\left|a_{4}\right|^{2}+\left|a_{6}\right|^{2}+\left|a_{7}\right|^{2}\right) \\
& +\left|a_{2}\right|^{2}\left(\left|a_{4}\right|^{2}+\left|a_{5}\right|^{2}+\left|a_{7}\right|^{2}\right) \\
& +\left|a_{3}\right|^{2}\left(\left|a_{4}\right|^{2}+\left|a_{5}\right|^{2}+\left|a_{6}\right|^{2}\right) \\
& -2 \operatorname{Re}\left(a_{0} a_{5} a_{1}^{*} a_{4}^{*}+a_{0} a_{6} a_{2}^{*} a_{4}^{*}\right. \\
& +a_{0} a_{7} a_{3}^{*} a_{4}^{*}+a_{1} a_{6} a_{2}^{*} a_{5}^{*} \\
& \left.+a_{1} a_{7} a_{3}^{*} a_{5}^{*}+a_{2} a_{7} a_{3}^{*} a_{6}^{*}\right), \\
& \\
\operatorname{Det}\left(\rho_{2}\right) & =\left|a_{0}\right|^{2}\left(\left|a_{3}\right|^{2}+\left|a_{6}\right|^{2}+\left|a_{7}\right|^{2}\right) \\
& +\left|a_{1}\right|^{2}\left(\left|a_{2}\right|^{2}+\left|a_{6}\right|^{2}+\left|a_{7}\right|^{2}\right) \\
& +\left|a_{4}\right|^{2}\left(\left|a_{4}\right|^{2}+\left|a_{3}\right|^{2}+\left|a_{7}\right|^{2}\right) \\
& +\left|a_{5}\right|^{2}\left(\left|a_{4}\right|^{2}+\left|a_{3}\right|^{2}+\left|a_{6}\right|^{2}\right) \\
& -2 \operatorname{Re}\left(a_{0} a_{3} a_{1}^{*} a_{2}^{*}+a_{0} a_{6} a_{2}^{*} a_{4}^{*}\right. \\
& +a_{0} a_{7} a_{2}^{*} a_{5}^{*}+a_{1} a_{6} a_{3}^{*} a_{4}^{*} \\
& \left.+a_{1} a_{7} a_{3}^{*} a_{5}^{*}+a_{4} a_{7} a_{5}^{*} a_{6}^{*}\right),
\end{aligned}
$$


and

$$
\begin{aligned}
\operatorname{Det}\left(\rho_{3}\right) & =\left|a_{0}\right|^{2}\left(\left|a_{3}\right|^{2}+\left|a_{5}\right|^{2}+\left|a_{7}\right|^{2}\right) \\
& +\left|a_{2}\right|^{2}\left(\left|a_{1}\right|^{2}+\left|a_{5}\right|^{2}+\left|a_{7}\right|^{2}\right) \\
& +\left|a_{4}\right|^{2}\left(\left|a_{1}\right|^{2}+\left|a_{3}\right|^{2}+\left|a_{7}\right|^{2}\right) \\
& +\left|a_{6}\right|^{2}\left(\left|a_{1}\right|^{2}+\left|a_{3}\right|^{2}+\left|a_{5}\right|^{2}\right) \\
& -2 \operatorname{Re}\left(a_{0} a_{3} a_{1}^{*} a_{2}^{*}+a_{0} a_{5} a_{1}^{*} a_{4}^{*}\right. \\
& +a_{0} a_{7} a_{1}^{*} a_{6}^{*}+a_{2} a_{5} a_{3}^{*} a_{4}^{*} \\
& \left.+a_{2} a_{7} a_{3}^{*} a_{6}^{*}+a_{4} a_{7} a_{5}^{*} a_{6}^{*}\right),
\end{aligned}
$$

we obtain:

$$
\begin{aligned}
\operatorname{Det}\left(\rho_{1}\right)-\operatorname{Det}\left(\rho_{2}\right)+ & \operatorname{Det}\left(\rho_{3}\right)=2\left(\left|a_{0}\right|^{2}\left|a_{5}\right|^{2}+\left|a_{1}\right|^{2}\left|a_{4}\right|^{2}\right. \\
& \left.+\left|a_{2}\right|^{2}\left|a_{7}\right|^{2}+\left|a_{3}\right|^{2}\left|a_{6}\right|^{2}\right) \\
& -2 \operatorname{Re}\left[a_{0} a_{7} a_{1}^{*} a_{6}^{*}+a_{2} a_{5} a_{3}^{*} a_{4}^{*}\right. \\
& \left.-\left(a_{0} a_{7}-a_{1} a_{6}\right)\left(a_{2}^{*} a_{5}^{*}-a_{3}^{*} a_{4}^{*}\right)\right] \\
& -4 \operatorname{Re}\left(a_{0} a_{5} a_{1}^{*} a_{4}^{*}+a_{2} a_{7} a_{3}^{*} a_{6}^{*}\right) .
\end{aligned}
$$

Given the time-optimal unitary evolution (30), we can compute the following nonzero time-dependent amplitudes (all modulo $\exp [-i \Omega \tau / 2]$ ):

S) representative of the class of fully separable initial states, $\left|\psi_{S}\right\rangle$ :

$$
\begin{aligned}
& \left(a_{S}\right)_{0}(\tau)=a_{1}^{*}, \\
& \left(a_{S}\right)_{2}(\tau)=-i \hat{B}_{0} e^{i \theta} s_{1} ;
\end{aligned}
$$

B1) representative of the class of bi-separable initial states, $\left|\psi_{B 1}\right\rangle$ :

$$
\begin{aligned}
& \left(a_{B 1}\right)_{0}(\tau)=-i \frac{\hat{B}_{0}}{\sqrt{2}} e^{-i \theta_{0}} s_{1}, \\
& \left(a_{B 1}\right)_{1}(\tau)=\frac{a_{2}^{*}}{\sqrt{2}} \\
& \left(a_{B 1}\right)_{2}(\tau)=\frac{e^{i \hat{\Omega} \tau}}{\sqrt{2}} a_{1} \\
& \left(a_{B 1}\right)_{3}(\tau)=-i \frac{\hat{B}_{0}}{\sqrt{2}} e^{i \theta} s_{2}
\end{aligned}
$$

B2) representative of the class of bi-separable initial states, $\left|\psi_{B 2}\right\rangle$ :

$$
\begin{aligned}
& \left(a_{B 2}\right)_{1}(\tau)=\frac{a_{2}^{*}}{\sqrt{2}}, \\
& \left(a_{B 2}\right)_{3}(\tau)=-i \frac{\hat{B}_{0}}{\sqrt{2}} e^{i \theta} s_{2}, \\
& \left(a_{B 2}\right)_{4}(\tau)=\frac{a_{3}^{*}}{\sqrt{2}}, \\
& \left(a_{B 2}\right)_{6}(\tau)=-i \frac{\hat{B}_{0}}{\sqrt{2}} e^{i \theta} s_{3} ;
\end{aligned}
$$

B3) representative of the class of bi-separable initial states, $\left|\psi_{B 3}\right\rangle$ :

$$
\begin{aligned}
& \left(a_{B 3}\right)_{0}(\tau)=-i \frac{\hat{B}_{0}}{\sqrt{2}} e^{-i \theta_{0}} s_{1}, \\
& \left(a_{B 3}\right)_{2}(\tau)=\frac{e^{i \hat{\Omega} \tau}}{\sqrt{2}} a_{1}, \\
& \left(a_{B 3}\right)_{4}(\tau)=\frac{a_{3}^{*}}{\sqrt{2}} \\
& \left(a_{B 3}\right)_{6}(\tau)=-i \frac{\hat{B}_{0}}{\sqrt{2}} e^{i \theta} s_{3}
\end{aligned}
$$

$\mathrm{W})$ representative of the class of $W$ initial states, $\left|\psi_{W}\right\rangle$ :

$$
\begin{aligned}
& \left(a_{W}\right)_{0}(\tau)=-i \frac{\hat{B}_{0}}{\sqrt{3}} e^{-i \theta_{0}} s_{1}, \\
& \left(a_{W}\right)_{1}(\tau)=\frac{a_{2}^{*}}{\sqrt{3}} \\
& \left(a_{W}\right)_{2}(\tau)=\frac{e^{i \hat{\Omega} \tau}}{\sqrt{3}} a_{1} \\
& \left(a_{W}\right)_{3}(\tau)=-i \frac{\hat{B}_{0}}{\sqrt{3}} e^{i \theta} s_{2}, \\
& \left(a_{W}\right)_{4}(\tau)=\frac{a_{3}^{*}}{\sqrt{3}} \\
& \left(a_{W}\right)_{6}(\tau)=-i \frac{\hat{B}_{0}}{\sqrt{3}} e^{i \theta} s_{3}
\end{aligned}
$$

GHZ) representative of the class of GHZ initial states, $\left|\psi_{G H Z}\right\rangle$ :

$$
\begin{aligned}
& \left(a_{G H Z}\right)_{0}(\tau)=\frac{a_{1}^{*}}{\sqrt{2}}, \\
& \left(a_{G H Z}\right)_{2}(\tau)=-i \frac{\hat{B}_{0}}{\sqrt{2}} e^{i \theta} s_{1}, \\
& \left(a_{G H Z}\right)_{5}(\tau)=-i \frac{\hat{B}_{0}}{\sqrt{2}} e^{-i \theta_{0}} s_{4}, \\
& \left(a_{G H Z}\right)_{7}(\tau)=\frac{e^{i \hat{\Omega} \tau}}{\sqrt{2}} a_{4} .
\end{aligned}
$$


[1] A. Einstein, B. Podolsky and N. Rosen, Phys. Rev. 47, 777 (1935).

[2] C. H. Bennett, G. Brassard, C. Crepeau, R. Jozsa, A. Peres, and W.K. Wootters, Phys. Rev. Lett. 70, 1895 (1993).

[3] C. H. Bennett and S. J. Wiesner, Phys. Rev. Lett. 69, 2881 (1992).

[4] A. Ekert, Phys. Rev. Lett.67, 661 (1991).

[5] M. Beck, Phys. Rev. Lett.84, 5748 (2000).

[6] N. Sangouard, C. Simon, H. De Riedmatten and N. Gisin, Rev. Mod. Phys.83, 33 (2011).

[7] V. Giovannetti, S. Lloyd and L. Maccone, Phys. Rev. Lett.96, 010401 (2006).

[8] L. Amico, R. Fazio, A. Osterloh and V. Vedral, Rev. Mod. Phys.80, 517 (2008).

[9] L. Borsten, D. Dahanayake, M.J. Duff, H. Ebrahim and W. Rubens, Phys. Rep. 471, 113 (2009).

[10] M.A. Nielsen and I.L. Chuang, Quantum Computation and Quantum Information (Cambridge University Press, Cambridge, 2000).

[11] R. Horodecki, P. Horodecki, M. Horodecki and K. Horodecki, Rev. Mod. Phys. 81, 865 (2009).

[12] I. Bengtsson and K. Zyczkowski, Geometry of Quantum States: an Introduction to Quantum Entanglement (Cambridge University Press, Cambridge, 2006).

[13] N. Margolus and L.B. Levitin, Physica D120, 188 (1998).

[14] L.B. Levitin and T. Toffoli, Phys. Rev. Lett. 99, 110502 (2007).

[15] V. Giovannetti, S. Lloyd and L. Maccone, Europhys. Lett. 62, 615 (2003)

[16] V. Giovannetti, S. Lloyd and L. Maccone, Phys. Rev. A67, 052109 (2003).

[17] J. Batle, M. Casas, A.R. Plastino and A. Plastino, Phys. Rev. A72, 032337 (2005).

[18] J. Batle, A. Borras, M. Casas, A.R. Plastino and A. Plastino, Phys. Rev. A82, 056302 (2010).

[19] A. Borras, M. Casas, A.R. Plastino and A. Plastino, Phys. Rev. A74, 022326 (2006).

[20] C. Zander, A.R. Plastino, A. Plastino and M. Casas, J. Phys. A40, 2861 (2007)

[21] J. Kupferman and B. Reznik, Phys. Rev. A78, 042305 (2008).

[22] C. Brif., R. Chakrabarti and H. Rabitz, New J. Phys. 12, 075008 (2010).

[23] S. Glaser, U. Boscain, T. Calarco, C. Koch, W. Kockenberger, R. Kosloff, I. Kuprov, B. Luy, S. Schirmer, T. Schulte-Herbruggen, D. Sugny and F. Wilhelm, Eur. Phys. J. D69, 279 (2015).

[24] A. Carlini, A. Hosoya, T. Koike and Y. Okudaira, Phys. Rev. Lett. 96, 060503 (2006).

[25] From the Greek " $\beta \rho \alpha \chi \iota \sigma \tau o \zeta$ ", i.e., fast, and " $\chi \rho о \nu o \zeta$ ", i.e time.

[26] A. Carlini, A. Hosoya, T. Koike and Y. Okudaira, J. Phys. A41, 045303 (2008).

[27] A. Carlini, A. Hosoya, T. Koike and Y. Okudaira, Phys. Rev. A75, 042308 (2007).

[28] A. Carlini and T. Koike, J. Phys. A46, 045307 (2013).

[29] A. Carlini and T. Koike, Phys. Rev. A86, 054302 (2012).

[30] T. Koike and Y. Okudaira, Phys. Rev. A 82, 042305 (2010).

[31] X. Wang, M. Allegra, K. Jacobs, S. Lloyd, C. Lupo and
M. Mohseni, Phys. Rev. Lett. 114, 170501 (2015).

[32] B. Russell and S. Stepney, J. Phys. A48, 115303 (2015).

[33] J. Geng, Y. Wu, X. Wang, K. Xu, F. Shi, Y. Xie, X. Rong and J. Du, Phys. Rev. Lett. 117, 170501 (2016).

[34] M. Bina, F. Casagrande, M. Genoni, A. Lulli and M. Paris, Eur. Phys. Lett. 90, 30010 (2010).

[35] Z.X. Man, Y.J. Zhang, F. Su and Y.J. Xia, Eur. Phys. J. D58, 147 (2010).

[36] M. Dukalski and Y.M. Blanter, J. Phys. B45, 245504 (2012).

[37] J.G. Amaro and C. Pineda, Phys. Scr. 90, 068019 (2015).

[38] I.M. Mirza, arXiv:1503.06930

[39] N. Behzadi, B. Ahansaz and S. Kazemi, Int. J. Theor. Phys. 55, 1577 (2016).

[40] F. Buscemi and P. Bordone, Phys. Rev. A87, 042310 (2013).

[41] A.P. Majtey and A.R. Plastino, Int. J. Quantum Inf.10, 1250063 (2012).

[42] M. Ali, Phys. Lett. A378, 2048 (2014).

[43] G. Morigi, J. Eschner, C. Cormick, Y. Lin, D. Liebfried and D.J. Wineland, Phys. Rev. Lett. 115, 200502 (2015).

[44] D. Park, Quantum Inf. Process. 15, 3189 (2016).

[45] J.K. Kalaga, A. Kowalewska-Kudlaszyk, W. Leonski and A. Barasinski, Phys. Rev. A94, 032304 (2016).

[46] X.W. Xu, Y.J. Zhao and Y.X. Liu, Phys. Rev. A88, 022325 (2013).

[47] Y.H. Kang, Y.H. Chen, Z.C. Shi, J. Song and Y. Xia, Phys. Rev. A94, 052311 (2016).

[48] Y.H. Chen, Y. Xia, J. Song and B.H. Huang, Opt. Comm. 380, 140 (2016).

[49] Y. Dai, Z. Shen and Y. Shi, J.H.E.P. 09, 071 (2015).

[50] Y. Dai, Z. Shen and Y. Shi, Phys. Rev. D94, 025012 (2016).

[51] A. Valdes-Hernandez, A.P. Majtey and A.R. Plastino, Phys. Rev. A91, 032313 (2015).

[52] F. Ticozzi and L. Viola, Quant. Inf. and Comp. 14, 0265 (2014).

[53] S.Y. Huang, H.S. Goan, X.Q. Li and G..J. Milburn, Phys. Rev. A88, 062311 (2013).

[54] K. Jacobs, R. Wu, X. Wang, S. Ashhab, Q.M. Chen and H. Rabitz, Eur. Phys. Lett. 114, 40007 (2016).

[55] J.T. Stockburger, R. Schmidt and J. Ankerhold, Phys. Scr. T165, 015020 (2015).

[56] M. Otten, J. Larson, M. Min, S.M. Wild, M. Pelton and S.K. Gray, Phys. Rev. A94, 022312 (2016).

[57] T. Pichler, T. Caneva, S. Montangero, M.D. Lukin and T. Calarco, Phys. Rev.A93, 013851 (2016).

[58] A. Borras, C. Zander, A.R. Plastino, M. Casas and A. Plastino, Europhys. Lett. 81, 300007 (2008).

[59] A. Borras, C. Zander, A.R. Plastino, M. Casas and A. Plastino, Phys. Rev. A78, 052104 (2008).

[60] A. Borras, A.P. Majtey and M. Casas, Phys. Rev. A78, 022328 (2008).

[61] B.K. Zhao, F.G. Deng, F.S. Zhang and H.Y. Zhou, Phys. Rev. A80, 052106 (2009).

[62] N. Khaneja, B. Heitmann, A. Spörl, H. Yuan, T. SchulteHerbrüggen, and S.J. Glaser, Phys. Rev. A75, 012322 (2007).

[63] H. Yuan, S.J. Glaser and N. Khaneja, Phys. Rev. A76, 012316 (2007). 
[64] H. Yuan, R. Zeier and N. Khaneja, Phys. Rev. A77, 032340 (2008).

[65] A. Carlini, A. Hosoya, T. Koike and Y. Okudaira, J. Phys. A44, 145302 (2011).

[66] M. Nimbalkar, R. Zeier, J.L. Neves, S.B. Elavarasi, H. Yuan, N. Khaneja, K. Dorai and S.J. Glaser, Phys. Rev. A85, 012325 (2012).

[67] H. Yuan, D. Wei, Y. Zhang, S. Glaser and N. Khaneja, Phys. Rev. A89, 042315 (2014).

[68] L. Van Damme, R. Zeier, S.J. Glaser and D. Sugny, Phys. Rev. A90, 013409 (2014).

[69] N. Khaneja and S.J. Glaser, Chem. Phys. 267, 11 (2001).

[70] N. Khaneja, R. Brockett and S.J. Glaser, Phys. Rev. A63, 032308 (2001).

[71] V. Coffman, J. Kundu and W.K. Wootters, Phys. Rev. A 61, 052306 (2000).

[72] W. Dür, G. Vidal and I. Cirac, Phys. Rev. A62, 062314 (2000).

[73] H. Ollivier and V.H. Zurek, Phys. Rev. Lett. 88, 017901 (2001).

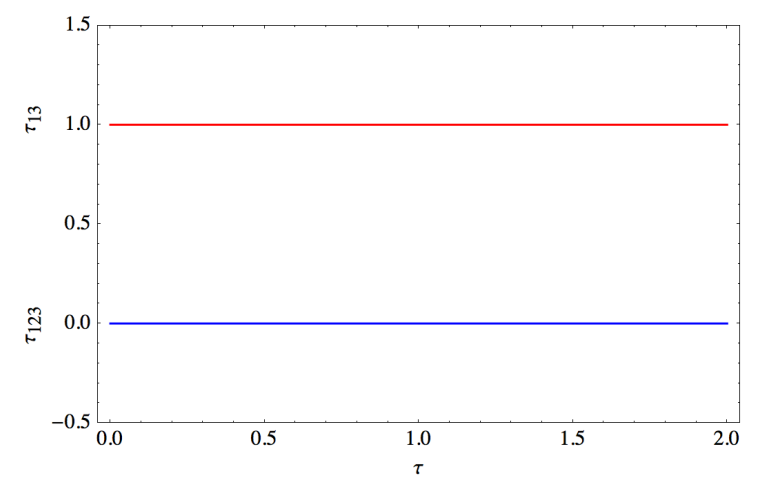

FIG. 1: The 2-tangle (red curve) and the 3-tangle (blue curve) as a function of time for the $B 2$ initial states and the coupling ratio $K=1$.

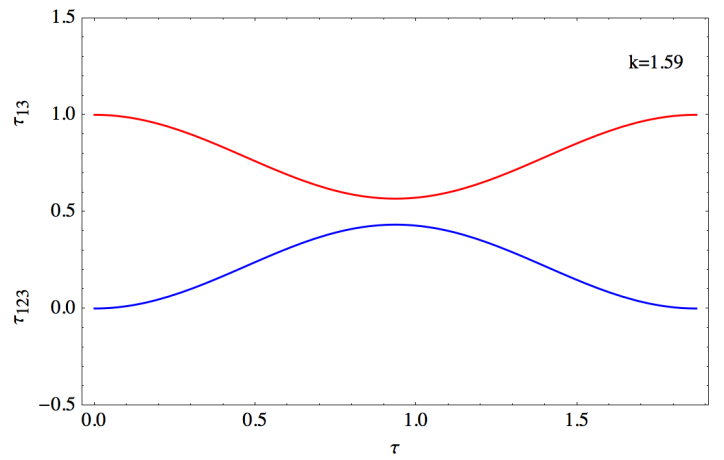

FIG. 2: The 2-tangle (red curve) and the 3-tangle (blue curve) as a function of time for the $B 2$ initial states and the coupling ratio $K=1.59$.

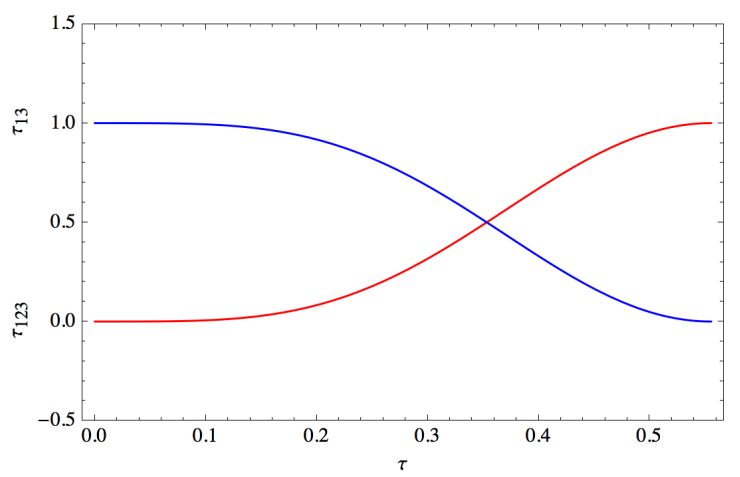

FIG. 3: The 2-tangle (red curve) and the 3-tangle (blue curve) as a function of time for the $G H Z$ initial states and the coupling ratio $K=1$.

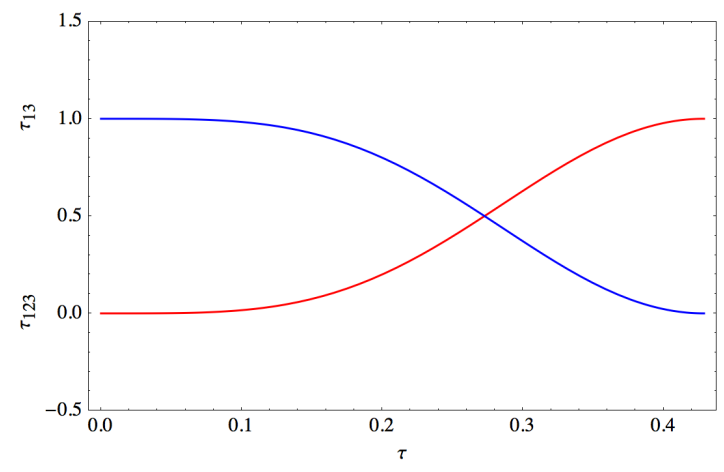

FIG. 4: The 2-tangle (red curve) and the 3-tangle (blue curve) as a function of time for the $G H Z$ initial states and the coupling ratio $K=1.59$. 